\title{
On the requirement for a high resolution spectrograph to investigate the multiplicity of massive stars with the 3.6-m Devasthal Optical Telescope
}

\author{
Michaël De Becker*
}

\author{
Space sciences, Technologies and Astrophysics Research (STAR) Institute, University of Liège, \\ Quartier Agora, 19c, Allée du 6 Août, B5c, B-4000 Sart Tilman, Belgium
}

\begin{abstract}
The investigation of the multiplicity of massive stars constitutes a key aspect of their understanding. On the one hand, the evolution of short period systems can be tremendously influenced by the companion, for instance through mass exchange or common envelope evolution. On the other hand, the wind-wind interaction region in massive multiple systems is the seat of a high level physics, including strong thermal X-ray emission, particle acceleration and non-thermal emission processes. The description and understanding of these processes require the accurate determination of orbital parameters, notably through spectroscopic studies. In this context, it is timely to anticipate some relevant applications of a high resolution spectrograph (HRS) to be mounted on the Devasthal Optical Telescope, emphasizing its potential complementarity with other Indian facilities such as the GMRT and Astrosat.
\end{abstract}

Keywords: Massive stars - Spectroscopy - Binaries - Visible

\section{A few biases which severely affect multiplicity investigations}

The identification of binaries, especially among massive stars, is affected by several biases. This must be kept in mind when one wants to define clearly whether a massive star has companions or not. These biases are related to the following factors:

- Orientation of the system: The ideal orientation to reveal a binary motion differs with the technique. Radial velocity (RV) variations investigated using a HRS can be measured on spectral lines for not too low inclinations. Depending on the inclination of the system, RV measurements may be (or not) the most adequate technique. A HRS would be adequate only in cases where RV curves could be built with a significant accuracy. If the inclination is too low, astrometric techniques such as long baseline optical/infrared interferometry (see e.g. Le Bouquin et al.2017) should be favored, provided the angular separation is compliant with the angular resolution of the interferometer which is typically a few milli-arcsecond (mas).

- Time sampling: Multiplicity investigations require a lot of observing time to sample the orbit. For long period orbits, observation campaigns can be quite long, and potentially difficult to or-

*Michael.DeBecker@uliege.be 
ganize. In addition, eccentric systems may display strong variations close to periastron passage only, with a flatter RV curve at other orbital phases. In the latter case, a poor time sampling may not cover the short orbital phase interval where RVs change above the detection limit.

- Period range: Very long period systems (typically more than $\sim 10$ years) are difficult to study because of the long duration of the required time basis, and more important because of the low amplitude of the RV variations which fall below the detection limit (typically not better than several $\mathrm{km} / \mathrm{s}$ because of the large rotational broadening of massive star spectral lines). For such long periods, astrometric techniques are more appropriate. As a rule of thumb, a separation of 1 Astronomical Unit (AU) at $1 \mathrm{kpc}$ converts into an angular separation of 1 mas. Separations of a few AU are expected for massive star systems with periods of a few years. Interestingly enough, there is an overlap between the range of periods likely to be studied using spectroscopy and interferometry, i.e. in the $1-10 \mathrm{yr}$ range (for systems at $1-2 \mathrm{kpc}$ ). In this domain, both techniques may be combined to reconstruct the full 3D orbit of the system.

\section{Some good reasons to mount a HRS on the DOT}

A key aspect of the investigation of multiple systems is the organization of campaigns with repeated observations of the same target. Opening the access to a telescope whose scheduling is much less constrained than for most major observatories (i.e. Very large Telescopes at the European Southern Observatory) is especially interesting. Repeated observations with high over-subscription factor telescopes are most of the time difficult to organize, preventing thus long term multiplicity studies to be accomplished. In addition, the improvement of the capability to investigate Northern sources with a 3.6-m telescope is also an asset. Not so many telescopes of that size exist in the Northern hemisphere, and the few existing ones are often affected by a restricted access for the international community. Finally, the size of the DOT is especially well adapted for the study a massive stars up to a few kpc, with good resolving power $(\mathrm{R} \sim 30000-50000)$ and high signal-to-noise ratio $(>100)$ spectra in a rather short exposure time (less than 1 hour). This would allow one to monitor such massive systems and collect high quality data with a reasonable cost in term of exposure time.

\section{Scientific questions that would benefit of a HRS on the DOT}

\subsection{The binary fraction among massive stars}

One knows that a significant fraction $(>50 \%)$ of massive stars are at least binary systems. A better determination of that fraction is very important to constrain stellar formation scenarios. In addition, it is clear that binarity influences significantly the evolution of massive stars (Sana et al.2012). One may for instance mention mass and common envelope evolution (Taam \& Sandquist 2000).

\subsection{Particle acceleration and non-thermal physics in massive binaries}

A fraction of massive binaries are known to accelerate particles up to relativistic energies. This process is revealed mainly by the identification of synchrotron radio emission in about 40 systems (De Becker \& Raucq 2013), along with non-thermal X-ray and gamma-ray emission at least in the case of Eta Car (Farnier et al. 2011, Hamaguchi et al. 2018). See De Becker (2018, 1st BINA workshop proceedings) for a general discussion.

As these processes occur in the colliding-wind region, they vary as a function of the orbital phase, and it is crucial to know as much details as possible to discuss that physics in the appropriate context. 
The use of high quality spectroscopic data collected with the DOT would help to derive accurate orbital elements, which is required to model these environments. Such observations may complement GMRT campaigns dedicated to these objects (see e.g. Ishwara-Chandra et al.2019, De Becker et al. 2019, in these proceedings). In addition, a crucial question at the centre of this field is related to the fraction of particle accelerator among massive binaries (De Becker et al. 2017). Addressing this question requires to improve (i) our knowledge of the multiplicity of massive stars, in parallel with (ii) the search for non-thermal signatures in their radio emission. The former requirement can be fulfilled notably by the monitoring of massive stars using a HRS mounted on the DOT.

\subsection{Thermal X-ray emission from massive binaries}

When the stellar winds of massive stars collide in a binary system, strong shocks are produced. The post-shock gas is heated up to temperatures of a few $10^{7} \mathrm{~K}$, and such a hot plasma produces thermal X-ray emission (Stevens et al. 1992, Pittard \& Parkin 2010). The detailed investigation of this X-ray emission allows one to study the hydrodynamics of the shocks.

The measured X-ray spectrum produced in the wind-wind interaction region is phase-locked with the orbital motion (see e.g. De Becker et al.2006, Pandey et al.2014, Arora \& Pandey 2018). On the one hand, the emission process depends on the stellar separation which is varying in eccentric systems. On the other hand, the stellar wind material is responsible for a significant photoelectric absorption that depends on the line of sight. This is clearly an orientation effect, whose modelling requires an accurate description of the orbit of the system. In this context, combined observations involving the DOT and Astrosat could be envisaged.

\subsection{Fundamental parameters of massive stars}

Using stellar spectroscopy to study the orbital motion of massive stars leads to the determination of their minimum masses. When a complementary technique can be used to determine the inclination of the system, absolute masses can be obtained. This is for instance the purpose of combined interferometric and spectroscopic measurements (Sana et al. 2013, Le Bouquin et al. 2017). Absolute masses constitute key parameters to understand stellar evolution.

On the other hand, the inspection of the spectral properties of stars in a multiple system is very important to infer their spectral type and luminosity classes. The use of disentangling techniques may even allow to efficiently separate spectra and confront them to stellar atmosphere models to determine various parameters including element abundances (e.g. Mahy et al. 2017). The use of these techniques requires the availability of high quality spectral time series covering the orbit.

\subsection{Production of dust particles in WC-type binaries}

Among the most evolved counterparts of massive stars, WC-type stars produce very dense stellar winds. A noticeable feature of these winds is the significant enrichment in carbon, which is the signature of the triple- $\alpha$ process It is referred to Crowther (2017) for a comprehensive review on Wolf-Rayet stars. When a WC wind is compressed because of a collision with the companion's wind in a binary system, the wind collision region gas reaches a density high enough for the nucleation of dust particles (Williams 2014). This phenomenon is at the origin of the so-called pinwheel nebulae revealed by infrared imaging (Tuthill et al.2008). The knowledge of orbital elements is a prerequisite for any attempt to model such a dust production process. 


\section{Concluding remarks}

Less than 20 years ago, the main paradigm about multiplicity of massive stars was to consider that they were a priori to be viewed as single objects, unless some evidence for binarity could be established for a fraction of them. Now, it becomes more and more clear that massive stars are very frequently found in binary systems. As our census of multiplicity of massive stars makes progress, one may even think about switching to a different paradigm where a significant fraction of massive stars are found in triple, or even higher multiplicity systems. Considering the strong influence of observational biases for multiplicity investigations of massive stars, a huge observational efforts using adequate tools is strongly needed.

Considering the high importance of binarity - and even higher multiplicity - of massive stars, observational studies contributing to improve (i) the census of stellar companions and (ii) the determination of orbital parameters are more than welcome. In this context, a High Resolution Spectrometer to be mounted on the 3.6-m Devasthal Optical Telescope would constitute a highly adequate opportunity, especially considering the complementarity with other techniques at other wavebands used to investigate other aspects of their physics in connection with binarity. In modern astrophysics, adopting multi-wavelength and multi-technique approaches constitutes the key to investigate in-depth the nature and the behaviour of astrophysical environments. In this context, multiplicity investigations of massive stars are not an exception, and some significant room for such an instrument exists in the present astronomical landscape.

\section{Acknowledgements}

The author would like to warmly thank the organizers for a fruitful and constructive workshop, along with BINA PIs and founders for pushing forward to make BINA such a promising initiative.

\section{References}

Arora B., Pandey J. C. 2018, BSRSL, 87, 193

Crowther P. A. 2007, ARA\&A, 45, 177

De Becker M, Rauw G., Sana H. et al. 2006, MNRAS, 371, 1280

De Becker M., Raucq F. 2013, A\&A, 558, A28

De Becker M., Benaglia P., Romero G. E., Peri C. S. 2017, A\&A, 600, A47

De Becker M. 2018, BSRSL, 87, 185

De Becker M., Ishwara-Chandra C. H., Benaglia P. 2019, BSRSL, 88, 262 (this volume)

Farnier C., Walter, R., Leyder J.-C. 2011, A\&A, 526, A57

Hamaguchi K., Corcoran M. F., Pittard J. M. et al. 2018, NatAs, 2, 731

Ishwara-Chandra C. H., Benaglia P., De Becker M., Tej A. 2019, BSRSL, 88, 166 (this volume)

Le Bouquin J.-B., Sana H., Gosset E. et al. 2017, A\&A, 601, A34

Mahy L., Damerdji Y., Gosset E. et al. 2017, A\&A, 607, A96

Pandey J. C., Pandey S. B., Karmakar S. 2014, ApJ, 788, 84

Pittard J. M., Parkin E. R. 2010, MNRAS, 403, 1657

Sana H., de Mink S. E., de Koter A. et al. 2012, Sci, 337, 444

Sana H., Le Bouquin J.-B., Mahy L. 2013, A\&A, 553, A131

Stevens I. R., Blondin J. M., Pollock A. M. T. 1992, ApJ, 386, 265

Taam R. E., Sanquist E. L. 2000, ARA\&A, 38, 113

Tuthill P. G., Monnier J. D., Lawrance N. et al. 2008, ApJ, 675, 698

Williams P. M. 2014, MNRAS, 445, 1253 